\title{
Systematic Transcriptome Analysis of Synovial Sarcoma Reveals Novel Transcriptional And Post- Transcriptional Abnormalities
}

\section{Zhengwang Sun}

Fudan University Shanghai Cancer Center

\section{Qiyue Xu}

East China Normal University

\section{Quan Huang}

Shanghai Changzheng Hospital

\section{Mengchen Yin}

Shanghai University of Traditional Chinese Medicine

\section{Hongqiang Zhang}

Fudan University Shanghai Cancer Center Minhang Branch

\section{Geng Chen ( $\square$ gchen@bio.ecnu.edu.cn )}

East China Normal University

\section{Yong Chen}

Fudan University Shanghai Cancer Center

\section{Wangjun Yan}

Fudan University Shanghai Cancer Center

\section{Research Article}

Keywords: synovial sarcoma, gene expression, alternative splicing, gene fusion, circular RNA

Posted Date: September 16th, 2021

DOI: https://doi.org/10.21203/rs.3.rs-852122/v1

License: (1) (1) This work is licensed under a Creative Commons Attribution 4.0 International License. Read Full License 


\section{Abstract}

Background: Synovial sarcoma (SS) is a rare and aggressive cancer that can come from distinct soft tissue types including muscle and ligaments. The SS transcriptome is crucial for understanding the SS biology; however, the transcriptomic landscape of SS is still poorly understood.

Methods: We performed deep total RNA sequencing on ten paired SS and tumor-adjacent tissues to systematically dissect the transcriptomic profile of SS in terms of gene expression, alternative splicing, gene fusion, and circular RNAs.

Results: A total of 2,309 upregulated and downregulated genes were identified between SS and tumoradjacent tissues. Those upregulated genes could lead to the upregulation of the cell cycle, ribosome, and DNA replication pathways, while the downregulated genes may result in the downregulation of a set of metabolic biological processes and signaling pathways. Moreover, 2,511 genes (including 21 splicing factors) were differentially alternative spliced, indicating that the deregulation of alternative splicing could be one important factor that contributes to the tumorigenesis. Additionally, we identified the known gene fusions of SS18-SSX1/SSX2 as well as 11 potentially novel gene fusions. Interestingly, 49 circular RNAs were differentially expressed and their parental genes could function in muscle contraction and muscle system processes.

Conclusions: Collectively, our comprehensive dissection of the transcriptomic profile of SS from both transcriptional and post-transcriptional levels provides novel insights into the biology and underlying molecular mechanism of SS.

\section{Introduction}

Synovial sarcoma (SS) is a rare and aggressive soft tissue cancer, which tends to occur near large joints, particularly in the extremities of the arms or legs, in young adults[1]. At present, surgery is still is the main treatment strategy for SS. Cytogenetically, a significant portion of SS cases involve nonrandom translocations between SS18 and SSX[2]. Although a range of studies has investigated the genetic profile of SS from different cascades, a comprehensive transcriptomic profile of SS from different aspects is still lacking[3, 4]. RNA sequencing (RNA-Seq) technologies provide unprecedented opportunities to gain insights into the transcriptome from various aspects, including expression level, alternative splicing (AS), gene fusions, and circular RNAs. These analyses are essential to systematically reveal and better understand the abnormally transcriptomic changes of SS; however, a comprehensive exploration of the SS transcriptome from these aspects is still currently lacking.

AS is a crucial mechanism of post-transcriptional modification responsible for increasing both transcriptome and proteome diversity of a cell in eukaryotes $[5,6]$. Since AS play important role in a variety of physiological processes (e.g. developmental programming), and the misregulation of AS can result in splicing defects which may have a pathogenic function to cause severe diseases, including cancers $[7,8]$. However, the genome-wide AS profile of SS is rarely studied to date. Furthermore, besides 
the common gene fusions formed by the translocation between chromosome $\mathrm{X}$ and 18 in SS, other gene fusions could also contribute to tumorigenesis or progression[9]. In addition, many circular RNAs (circRNAs) have been demonstrated to be functional as miRNA sponges and modulators of transcription[10,11], which could be vital for different aspects of malignant phenotypes, such as cell cycle, apoptosis, and invasion[12,13]. Moreover, some circRNAs are potentially important biomarkers for certain cancers[12,14,15]. But little is known about the expression profile of circRNAs in SS and almost no study has investigated this in SS. Thus, systematic dissection of the SS transcriptome from both transcriptional and post-transcriptional layers is necessary to better understand the underlying mechanisms of SS development.

Here we performed Ribo-Zero RNA-seq on ten pairs of Chinese SS and corresponding tumor-adjacent tissues to comprehensively explore the transcriptome profile of SS from various aspects. We first carried out differential expression calling and detected a number of upregulated and downregulated genes. Then the AS deregulation of a multitude of genes and a set of tumor-specific gene fusion events were identified. We also investigated the expression changes of circRNAs between SS and tumor-adjacent tissues. Moreover, we constructed an interaction network among circRNAs, miRNAs, and their target genes, which enabled us to further gain insights into the potential function of circRNAs in SS.

\section{Materials And Methods}

\section{RNA extraction}

Total RNA was extracted from the $10 \mathrm{mg}$ synovial sarcoma and tumor-adjacent tissues after grinding by Homogenizer (Scientz) using TRIzol® Reagent (Invitrogen) and RNeasy MinElute spin column (Qiagen) according to the manufacturer's instructions. Then the integrity of the total RNA was determined by 2100 Bioanalyser (Agilent) and quantified using the NanoDrop (Thermo Scientific). About 1 ug high-quality or media-quality RNA sample (OD260/280 $=1.9 \sim 2.0, \mathrm{RIN} \geq 4)$ was used to construct the sequencing library.

\section{Library preparation and RNA sequencing}

RNA purification, reverse transcription, library construction, and sequencing were performed at WuXi NextCODE in Shanghai according to the manufacturer's instructions (Illumina). The rRNA-depleted sequencing libraries from total RNA were prepared using Illumina TruSeq ${ }^{\circledR}$ Stranded Total RNA Gold preparation Kit. About 1 ug total RNA was used as input material, and then the Ribo-Zero Gold kit was used to remove both cytoplasmic and mitochondrial rRNA. After purification of the remaining RNA without rRNA, the RNA was fragmented into small pieces using divalent cations under elevated temperature. The cleaved RNA fragments are copied into the first-strand cDNA using reverse transcriptase and random primers, followed by second-strand cDNA synthesis. These cDNA fragments then were subjected to end-repair, phosphorylation, and 'A' base addition according to Illumina's library construction protocol. The products were purified and enriched with PCR, and the AMPure XP Beads (Beckmen) were used to clean up the amplified target fragments to create the final cDNA library. After library construction, Qubit 2.0 fluorometer dsDNA HS Assay (Thermo Fisher Scientific) was used to quantify the concentration 
of the resulting sequencing libraries, while the size distribution was analyzed using Agilent BioAnalyzer 2100 (Agilent).

Sequencing was performed using the Illumina system following Illumina-provided protocols for $2 \times 150$ paired-end sequencing in WuXi NextCODE at Shanghai, China.

\section{Short-read mapping and gene expression quantification}

The RNA-seq reads of each sample for 10 pairs of SS and tumor-adjacent tissues were separately aligned to the human reference genome GRCh38 using HISAT2[16] (version 2.1.0). Then we quantified the gene expression of each sample by employing StringTie[17] (version 1.3.6). The human gene annotation file in the GTF format of version 95 from the Ensembl database (http://www.ensembl.org) was used. The mapped read count and expression value in transcript per million (TPM) for each gene were obtained from StringTie and used for downstream analysis.

\section{Differential gene expression calling}

For differential expression analysis, the read count mapped to each gene was used as input. The gene expression changes between SS and tumor-adjacent tissues were examined using DESeq2[18] (version 1.24.0). We defined the differentially expressed genes (DEGs) with the threshold of |fold change| $>2$ and adjusted $P$-value $<0.01$.

\section{Detection of alternative splicing events}

We investigated the alternative splicing (AS) profile of genes between SS and tumor-adjacent tissues by employing rMATS[19] (version 4.0.2). The bam files outputted by HISAT2 after read mapping was used as the input. Five common AS types of exon skipping (ES), alternative $3^{\prime}$ acceptor sites (A3AS), alternative 5' donor sites (A5DS), intron retention (IE), and mutually exclusive exons (ME) were investigated. The differential alternative splicing events were identified with the cutoff of FDR $<0.05$.

\section{Identification of gene fusions}

In order to explore the genetic alterations, we employed TopHat-Fusion[20] (version 2.1.0) with default parameters to identify the gene fusion events in all tumor and normal samples. Only the fusions with at least 3 supporting reads and 2 supporting pairs were considered. Finally, 14 and 11 gene fusion pairs were detected in SS and tumor-adjacent tissues, respectively. We only kept the 14 gene fusions that are unique to SS and discarded the fusions detected in tumor-adjacent tissues.

\section{Circular RNA detection and differential expression analysis}

We investigated the expression profiles of circRNAs in SS and tumor-adjacent tissues using CIRI[21] (version 0.1.0). Then differential expression analysis was conducted by employing DESeq2 (version 1.24.0) based on the expression count of circRNAs identified by CIRI. Only the circRNAs with expression changes of |fold change| $>2$ and adjusted $P$-value $<0.01$ were considered as differentially expressed. The official IDs of circRNAs were obtained by coordinate mapping using the circBase[22] database. 


\section{Construction of interaction network among circRNAs, miRNAs, and target genes}

To gain insights into the function of circRNAs, we built an interaction network among the circRNAs, miRNAs, and the target genes of miRNAs. The PPI interactions were downloaded from the STRING[23] database (version 11.0). The regulatory relationship between miRNAs and target genes, as well as the known miRNA-circRNAs interactions, were obtained from the starBase[24] database (version 3.0). We only used the circRNA-miRNA pairs supported by $>5$ CLIP-seq experiments and the miRNA-target gene pairs supported by $>2$ CLIP-seq experiments and $>2$ degradome-seq experiments in the StarBase 2 database. Then we incorporated these interactions to construct the interaction network among circRNAs, miRNAs, and the genes targeted by miRNAs using Cytoscape[25] (version 3.7.2). Only the parental genes of differentially expressed circRNAs, DEGs, DASGs, and fusion genes were considered in the interaction network construction.

\section{Gene functional enrichment analysis}

We conducted gene ontology (GO) and KEGG pathway enrichment analyses using GSEA[26] (version 4.0.1) for the upregulated and downregulated DEGs between SS and tumor-adjacent tissues. The functional enrichment analysis of biological processes and pathways for the differentially alternative spliced genes, fusion genes, and the parental genes of circular RNAs were carried out with clusterProfiler[27] (version 3.12.0). The enriched items with adjusted $P$-value $<0.05$ were defined as significant.

\section{Results}

\section{An abundance of important genes are differentially expressed between SS and tumor-adjacent tissues}

To gain insights into the transcriptomic changes of SS patients, we deeply sequenced the tumor and tumor-adjacent tissues of 10 SS patients with total RNA sequencing (including both poly (A+) and poly (A-) RNAs). The detailed information and corresponding pathological diagnosis reports of these $10 \mathrm{SS}$ patients were shown in Table 1, respectively. We first aligned the RNA-seq reads of each sample to the human reference genome GRCh38 using HISAT2[16] and then conducted differential expression calling by employing DEseq2[18]. A total of 4,286 differentially expressed genes (DEGs) were detected using the threshold of |fold changel $>2$ and adjusted $P$-value $<0.01$, of which 2,309 (including 432 IncRNA genes) and 1,977 (including 333 IncRNA genes) genes were separately upregulated and downregulated in SS compared to tumor-adjacent tissues (Fig. 1A, Supplementary Table S1). Interestingly, we found that 340, 185,124 , and 7 of those DEGs are oncogenes, tumor suppressor genes (TSGs), transcription factors (TFs), and splicing factors (Supplementary Figure S1), respectively (Fig. 1B). Specifically, 52 TFs (such as AES and BCL6) were down-regulated and 72 TFs (e.g. ARID3A and BRCA2) were up-regulated, suggesting that the expression changes of these TFs could influence the expression of their downstream target 
genes including related oncogenes and TSGs. Since oncogenes and TSGs are closely correlated with cancer, their expression changes may play an important role in the development of SS. Specifically, in consideration of the crucial function of splicing factors in AS regulation[28], we further conducted a qPCR experiment to validate the expression profiles of the seven splicing factors (ELAVL2, HNRNPA1, HNRNPH2, MBNL1, PCBP1, QKI, and TIA1) in DEGs (Supplementary Figure S2). As expected, the experimental results were consistent with the RNA-seq data, indicating the robustness of our analysis. Therefore, the differential expression of these splicing factors could result in the AS deregulation of corresponding genes in SS.

Table 1

Detailed information of 10 synovial sarcoma patients.

\begin{tabular}{|lllllll|}
\hline $\begin{array}{l}\text { Patient } \\
\text { ID }\end{array}$ & Age & Sex & Tumor Location & Tumor Size $(\mathbf{c m})$ & Tumor status & Outcome \\
\hline 1 & 26 & Female & Thigh & $12 * 10.5 * 10$ & Primary & Alive \\
\hline 2 & 18 & Male & Foot & $6.0 * 3.5 * 2.0$ & Local recurrence & Died \\
\hline 3 & 37 & Male & Groin & $9.5 * 6 * 6$ & Local recurrence & Alive \\
\hline 4 & 29 & Female & Lung & $2.0 * 2.0 * 1.3$ & Primary & Alive \\
\hline 5 & 59 & Female & Iliac Bone & $5.5 * 4.5 * 3.5$ & Local recurrence & Alive \\
\hline 6 & 28 & Female & Foot & $2 * 1.7 * 0.7$ & Primary & Alive \\
\hline 7 & 20 & Female & Neck & $6 * 5 * 4$ & Primary & Alive \\
\hline 8 & 27 & Male & Shank & $7 * 6 * 2$ & Primary & Alive \\
\hline 9 & 41 & Male & Shank & $8 * 6 * 4$ & Local recurrence & Alive \\
\hline 10 & 71 & Female & Thigh & $9 * 6 * 3$ & Primary & Alive \\
\hline
\end{tabular}

Gene ontology (GO) and KEGG pathway enrichment analyses showed that these upregulated and downregulated DEGs were mainly involved in the fundamental and tumor-related biological processes and pathways (Fig. 1C, D, and E FDR < 0.05). For example, the up-regulated DEGs were primarily enriched in the cell-cycle-related biological processes (e.g. chromosome organization, chromatin organization, and DNA conformation change) and pathways of systemic lupus erythematosus, cell cycle, DNA replication, and P53 signaling (Fig. 1C). Several previous studies also identified the cell-cycle-related genes in sarcoma as a major category of up-regulated genes[29, 30], which was in line with our finding. By contrast, the down-regulated DEGs were mainly involved in the metabolic-related biological processes (such as energy derivation by oxidation of organic compounds, muscle system process, and glucan metabolic process) and the pathways of oxidative phosphorylation, insulin signaling pathway, and vascular smooth muscle contraction (Fig. 1D). Thus, the result suggests that a multitude of genes prominently altered their expression levels in SS, which could be one of the main factors accounting for tumorigenesis through up-regulating and down-regulating corresponding pathways. 


\section{Deregulation of alternative splicing could contribute to the tumorigenesis of SS}

Considering that the misregulation of AS can lead to the production of aberrant proteins that contribute to tumorigenesis[8], we further compared the AS profile between SS and tumor-adjacent tissues by employing rMATS[19]. Five classical splicing categories of exon skipping (ES), alternative 5' donor sites (A5DS), alternative $3^{\prime}$ acceptor sites (A3AS), mutually exclusive exons (ME), and intron retention (IR) were analyzed. In total, we identified 2511 (including 41 IncRNA genes) significantly differential AS genes (DASGs), of which 2018, 223, 242, 486, and 159 belong to the splicing mode changes of ES, A5DS, A3AS, $\mathrm{ME}$, and IR, respectively (Fig. 2A, FDR $<0.05$, Supplementary Table S2). As expected, ES was the most common differential splicing mode (80.37\%, 2018 out of 2511 DASGs), whereas IR was the least (6.33\%, 159 out of 2511 DASGs). Notably, the majority of those DASGs among the five classical splicing categories were largely different, only a small portion of them simultaneously exhibited three or more distinct splicing types (Fig. 2A).

Gene functional enrichment analysis indicated that those 2511 DASGs were mainly involved in the RNA splicing and cancer-related biological processes and KEGG pathways (Fig. 2B and C, adjusted $P$-value < 0.05), which was highly correlated with the AS process. For instance, the top enriched biological processes of those DASGs were mRNA processing, microtubule cytoskeleton organization, and RNA splicing, while the enriched pathways are endocytosis, RNA transport, proteoglycans in cancer, and spliceosome. Moreover, we observed that 21 splicing factor genes showed significantly differential AS between SS and tumor-adjacent tissues, such as HNRNPA1, PTBP2, QKI, RBFOX2, and TRA2A. It is well known that the splicing factors are crucial for AS regulation[28], the deregulation of those splicing factors could drastically disrupt the splicing process of many corresponding genes and contribute to the tumorigenesis of SS[31]. Furthermore, we found that 346, 204, and 122 oncogenes, TSGs, and TFs were also differentially spliced (Fig. 2D). The abnormal splicing of these TFs could influence the expression of their downstream target genes and contribute to the development and progression of SS. Only 368 genes shared between DASGs and DEGs, leaving most of them were distinct (Fig. 2E). These common 368 genes were enriched in the biological process of actomyosin structure organization and pathway of regulation of actin cytoskeleton (Fig. 2E, adjusted $P$-value $<0.05$ ). Thus, the genes that showed differential expression were quite distinct from those that exhibited differential splicing, suggesting that AS is complementary with expression level in revealing the transcriptomic changes. These results indicate that the abnormal AS changes of genes could be another important factor responsible for the tumorigenesis of SS.

\section{Dissection of the gene fusions in SS}

We further explored the gene fusion events in SS patients using TopHat-Fusion[20] A total of 14 and 11 unique gene fusion pairs were separately identified in SS and tumor-adjacent tissues, and no fusion was shared between them. The 14 tumor-specific gene fusion pairs were from seven SS patients, most of which (11 out of 14) were resulted from the rearrangements within the same chromosome, while 3 of 
them were generated by breaking and rejoining of two disparate chromosomes (Fig. 3A). In total, 27 genes were involved in these tumor-specific gene fusions. SS18 was fused with SSX1 and SSX2, which was in line with previous studies[9]. In constrast, other genes were mainly fused with one partner (Fig. 3B).

As shown in Fig. 3C, the maximum number of gene fusion pairs detected in individual patients was four and the gene fusion events in those SS patients were quite distinct. Intriguingly, these tumor-specific gene fusion events contain one TF of SSX2 and seven oncogenes of SS18, SSX1, SSX2, BCOR, CNOT1, HIST2H2AC, and TOP1 (Fig. 3D). Oncogene SS18 was fused with the TF and oncogene of SSX2 as well as the oncogene SSX1, which is consistent with the known findings[32]. Besides, other oncogenes of BCOR, CNOT1, HIST2H2AC, and TOP1 formed the fusion events of BCOR-CCNB3, CNOT1-SETD6, HIST2H2AC-HIST2H2AB, and TOP1-PLCG1-AS1, respectively. Previous studies have shown that BCORCCNB3 fusion tends to occur in the undifferentiated small round-cell sarcomas like Ewing sarcoma and has the potential to drive sarcoma progression[33-35]. Other gene fusions could be novel for SS, and the involved genes could be functionally important. For example, CNOT1 encodes the CCR4-NOT transcription complex subunit 1 , which mainly participates in deadenylating mRNAs[36]. HIST2H2AC and $\mathrm{HIST} 2 \mathrm{H} 2 \mathrm{AB}$ can generate the replication-dependent histones that are basic nuclear proteins responsible for the nucleosome structure of the chromosomal fiber. TOP1 encodes the enzyme of DNA topoisomerase for controlling and altering the topologic states of DNA during transcription[37]. Since TF could regulate the expression of many downstream target genes and oncogenes are closely associated with cancer, the fusion events of those TFs and oncogenes may contribute to the tumorigenesis/progression of SS. Interestingly, IncRNA genes of LINC00970, LOC105375787, and PLCG1AS1 were also involved in the gene fusion events, but their functions were still unknown. Gene functional enrichment analysis showed that those fusion genes were significantly enriched in the KEGG pathway of transcriptional misregulation in cancer (Fig. 3E, adjusted $P$-value $<0.05$ ).

Moreover, we further explored the expression profile of these fusion genes using synovial sarcoma data of The Cancer Genome Atlas (TCGA)[38]. As expected, these fusion genes showed similar expression patterns between the synovial sarcoma samples of us $(n=10)$ and TCGA $(n=7)$ (Supplementary Figure $S 3 A$ ). Additionally, we also found that these fusion genes exhibited slightly different expression profiles across distinct types of TCGA sarcomas (Supplementary Figure S3B). Considering that the number of synovial sarcoma samples is limited, we used all the TCGA sarcoma samples to do the survival analysis based on our identified fusion genes. Interestingly, the expression levels of KLRB1 and TOP1 were significantly associated with the survival of sarcoma patients (Fig. $3 F, P<0.05$ ), indicating that they could be potential prognostic markers.

\section{Circular RNAs may play a role in SS formation}

Emerging evidence shows that circRNAs can involve in various aspects of tumor biology[15, 39], thus we further investigated the expression profile of circRNAs in SS and tumor-adjacent tissues. We detected 49 differentially expressed circular RNAs by employing CIRI[21] with the threshold of |fold change| $>2$ and adjusted $P$-value $<0.01$. As shown in Fig. 4A, 21 of them were significantly up-regulated in SS, whereas 
the other 28 were down-regulated. Furthermore, we found that the great majority (46 out of $49,93.88 \%$ ) of those differentially expressed circRNAs were formed by the circulation of exons of their parental genes, only two circRNAs of 10:24380869|24384423 (parental gene: KIAA1217) and 17:35168061|35168685 (parental gene: UNC45B) were produced from the intronic region and another one (5:137757867|137759020) was generated in the intergenic region (Fig. 4B).

Intriguingly, 7, 5, and 3 of the parental genes for those differentially expressed circRNAs were oncogenes, TSGs, and TFs (Fig. 4C). Previous studies have shown that circRNAs can form posttranscriptional regulators to regulate the expression of their parental genes[40,41]. Thus, these circRNAs have the potential to affect the expression of their parental oncogenes, TSGs, and TFs. Gene functional enrichment analysis showed that the parental genes of those differentially expressed circRNAs were mainly enriched in the muscle system process (such as MAP2K4, HDAC4, TMEM38A, MYH1, MYH2, CAMK2G, TRDN, and SULF2) and muscle contraction (e.g. HDAC4, TMEM38A, MYH1, MYH2, TRDN, and SULF2) (Fig. 4D, adjusted $P$-value $<0.05$ ).

\section{The genes involved in different types of transcriptomic changes are largely distinct}

We further compared the four gene types of DEGs, DASGs, the fusion genes, and the parental genes of differentially expressed circRNAs. As shown in Fig. 5, the genes in one type were largely distinct from that of other types, and no genes were common among the four categories. Only a fraction of them was involved in two or three types of changes (Fig. 5). Intriguingly, the DEGs of BCOR, HIST2H2AB, and MEG8, and the DASGs of AKR1E2 and DCAF8 were overlapped with the fusion genes, suggesting that the fusion events may influence the expression and/or AS profile of these genes. BCOR is an oncogene, while MEG8 is an imprinted gene. Moreover, 18 DEGs (e.g. DNM30S, ZNF730, DNAH14, and AFF2) shared with the parental genes of differentially expressed circRNAs, implying that expression changes of these genes could affect the expression of circRNAs as well. In addition, 17 DASGs (such as SUCO, VWA8, MTUS1, and USP53) were common to the parental genes of differentially expressed circRNAs. Since circRNAs are mainly formed by AS of pre-mRNAs through backsplicing[42], the AS changes of these DASGs have the potential to influence the expression of corresponding circRNAs. Collectively, our results show that all the four transcriptomic aspects of expression changes, AS, gene fusions, and circRNAs could be closely correlated with the tumorigenesis/progression of SS.

\section{CircRNAs could potentially regulate the expression of a multitude of genes by acting as miRNA sponges}

An increasing number of studies suggested that endogenous circRNAs can act as miRNA sponges to regulate corresponding gene expression $[43,44]$. We further constructed the interaction network among differentially expressed circRNAs, miRNAs, and the miRNA target genes of DEGs, DASGs, and fusion genes to elucidate the functional roles of those differentially expressed circRNAs. Based on the known miRNA-circRNA regulations, and the miRNA-targets relationships in the starBase database[24] as well as the protein-protein interactions (PPIs) in the String database[23], the resulting interaction network involved in 5 circRNAs (hsa_circ_0001699, hsa_circ_0000247, hsa_circ_0000246, hsa_circ_0000095, and 
hsa_circ_0000118), 44 miRNAs, 293 protein-coding genes, containing 57 miRNA-circRNA interactions, 789 miRNA-mRNA interactions and 350 PPIs (Fig. 6).

It is well known that circRNAs can regulate gene expression by influencing transcription, mRNA turnover, and translation by sponging RNA-binding proteins (RBPs) and miRNAs[44]. Our resulting network showed that circRNAs hsa_circ_0001699, hsa_circ_0000247, hsa_circ_0000246, hsa_circ_0000095, and hsa_circ_0000118 could act as the sponges of 14, 13,13,12, and 5 miRNAs, respectively. Moreover, these miRNAs have the potential to regulate the expression of 119,202 , and 3 genes of DEGs, DASGs, and/or fusion genes. Base on the findings in previous studies[43, 44], the expression of these miRNA target genes could be indirectly influenced by corresponding circRNAs through competing for the interaction with miRNAs. Consequently, our result suggests that circRNAs could potentially function as miRNA sponges to regulate the expression of an abundance of corresponding genes.

\section{Discussion}

In this study, we systematically explored the transcriptome alterations of SS in terms of gene expression and AS, as well as gene fusions and circRNAs. A total of 4286 genes (including 765 IncRNA genes) were differentially expressed between SS and paired tumor-adjacent tissues, which were mainly involved in fundamental biological processes and cancer-related pathways. Moreover, we experimentally validated the differential expression of seven splicing factors using qPCR. We also detected 2511 genes (including 41 IncRNA genes) that showed differential AS, where the most common AS mode was ES $(80.37 \%$ of these DASGs), followed by ME, A3AS, A5DS, and IR. Gene functional enrichment analysis also showed that these DASGs were enriched in splicing related biological processes and pathways. Surprisingly, those DEGs and DASGs were largely distinct, only a small portion of them were the same, suggesting that AS is complementary with expression level for investigating transcriptomic changes. Notably, a fraction of those DEGs and DAGs were oncogenes, tumor suppressors, and TFs, indicating that they could be closely associated with the tumorigenesis of SS. Moreover, we identified 14 tumor-specific gene fusion pairs in SS, which not only included the known gene fusions of SS18-SSX[32] but also contained novel fusion events involving both protein-coding and IncRNA genes. Additionally, we observed that 49 circRNAs were markedly changed expression in SS compared to tumor-adjacent tissues, and their parental genes were enriched in the muscle system process.

To the best of our knowledge, we are the first to study the SS transcriptome from a comprehensive view covering both transcriptional and post-transcriptional levels. Specifically, the deregulation of AS and the role of circRNAs were rarely explored in SS previously. An increasing number of studies have shown that imbalances in the AS process can affect the development of various human diseases, especially the oncogenesis, progression, and metastasis of a range of cancers[45]. We identified 122 differentially spliced TFs and 124 differentially expressed TFs, suggesting that these TFs could be responsible for the expression level changes of an abundance of their target genes[46, 47]. Moreover, we observed that 7 and 21 splicing factors were dramatically changed in expression level or AS profile. Since splicing factors are essential in regulating the AS of genes, these abnormally changed splicing factors may significantly 
contribute to the AS changes of many related genes[48]. On the other hand, circRNAs have critical regulatory functions and play key roles in the initiation and progression of diverse diseases including cancers $[49,50]$. The differentially expressed circRNAs identified by us were mainly generated from the genes correlated with the muscle system process and contraction. We also constructed the interaction network among circRNAs, miRNAs, and downstream target genes to elucidate their potential regulatory mechanism. The resulting network indicated that those differentially expressed circRNAs have the potential to act as the sponge for dozens of miRNAs to indirectly regulate the expression of hundreds of DEGs and DASGs.

\section{Conclusions}

Collectively, we systematically dissected the transcriptomic profile of SS and identified a number of DEGs, DASGs, fusion genes, and circRNAs that could be closely associated with the tumorigenesis of SS. Our study not only gained novel insights into SS transcription and post-transcription but also shed light on the underlying molecular mechanisms.

\section{Declarations}

\section{Ethics approval and consent to participate}

Clinical samples were collected from Fudan University Shanghai Cancer Center of China. This study was approved by the Ethics Committee of Fudan University Shanghai Cancer Center. All patients in this study provided written informed consent for sample collection and data analyses as well as publication of identifying information in an online open-access publication. All methods were carried out in accordance with relevant guidelines and regulations.

\section{Consent for publication}

Not applicable.

\section{Availability of data and materials}

All raw and processed sequencing data generated in this study have been submitted to the NCBI Gene Expression Omnibus (GEO; https://www.ncbi.nlm.nih.gov/geo/) under accession number GSE144190.

\section{Competing interests}

Not applicable

\section{Funding}

This work was funded by the National Natural Science Foundation of China grant numbers 31771460 and 91629103. 
Author contributions

GC, YC and WY designed and supervised the study. ZS and HZ collected and prepared the samples. QX, $\mathrm{ZS}, \mathrm{QH}$, and MY performed the analysis. GC and ZS wrote the manuscript.

\section{Acknowledgments}

We thank the helpful discussion with Qizhi Li.

\section{References}

1. Ladanyi M, Antonescu CR, Leung DH, Woodruff JM, Kawai A, Healey JH, Brennan MF, Bridge JA, Neff JR, Barr FG et al: Impact of SYT-SSX fusion type on the clinical behavior of synovial sarcoma: a multi-institutional retrospective study of 243 patients. Cancer Res 2002, 62(1):135-140.

2. Przybyl J, Sciot R, Rutkowski P, Siedlecki JA, Vanspauwen V, Samson I, Debiec-Rychter M: Recurrent and novel SS18-SSX fusion transcripts in synovial sarcoma: description of three new cases. Tumour Biol 2012, 33(6):2245-2253.

3. Cancer Genome Atlas Research Network. Electronic address edsc, Cancer Genome Atlas Research N: Comprehensive and Integrated Genomic Characterization of Adult Soft Tissue Sarcomas. Cell 2017, 171(4):950-965 e928.

4. He RQ, Wei QJ, Tang RX, Chen WJ, Yang X, Peng ZG, Hu XH, Ma J, Chen G: Prediction of clinical outcome and survival in soft-tissue sarcoma using a ten-IncRNA signature. Oncotarget 2017, 8(46):80336-80347.

5. Wang ET, Sandberg R, Luo S, Khrebtukova I, Zhang L, Mayr C, Kingsmore SF, Schroth GP, Burge CB: Alternative isoform regulation in human tissue transcriptomes. Nature 2008, 456(7221):470-476.

6. Keren H, Lev-Maor G, Ast G: Alternative splicing and evolution: diversification, exon definition and function. Nat Rev Genet 2010, 11(5):345-355.

7. Wang GS, Cooper TA: Splicing in disease: disruption of the splicing code and the decoding machinery. Nat Rev Genet 2007, 8(10):749-761.

8. Zhang J, Manley JL: Misregulation of pre-mRNA alternative splicing in cancer. Cancer Discov 2013, 3(11):1228-1237.

9. Edwards PA: Fusion genes and chromosome translocations in the common epithelial cancers. $J$ Pathol 2010, 220(2):244-254.

10. Chen LL: The biogenesis and emerging roles of circular RNAs. Nat Rev Mol Cell Biol2016, 17(4):205-211.

11. Li X, Yang L, Chen LL: The Biogenesis, Functions, and Challenges of Circular RNAs. Mo/ Cell 2018, 71(3):428-442.

12. Greene J, Baird AM, Brady L, Lim M, Gray SG, McDermott R, Finn SP: Circular RNAs: Biogenesis, Function and Role in Human Diseases. Front Mol Biosci 2017, 4:38. 
13. Qu S, Yang X, Li X, Wang J, Gao Y, Shang R, Sun W, Dou K, Li H: Circular RNA: A new star of noncoding RNAs. Cancer Lett 2015, 365(2):141-148.

14. Abu N, Jamal R: Circular RNAs as Promising Biomarkers: A Mini-Review. Front Physiol 2016, 7.

15. Dong YP, He D, Peng ZZ, Peng W, Shi WW, Wang J, Li B, Zhang CF, Duan CJ: Circular RNAs in cancer: an emerging key player. J Hematol Oncol 2017, 10.

16. Kim D, Langmead B, Salzberg SL: HISAT: a fast spliced aligner with low memory requirements. Nat Methods 2015, 12(4):357-360.

17. Pertea M, Pertea GM, Antonescu CM, Chang TC, Mendell JT, Salzberg SL: StringTie enables improved reconstruction of a transcriptome from RNA-seq reads. Nature Biotechnology 2015, 33(3):290-+.

18. Love MI, Huber W, Anders S: Moderated estimation of fold change and dispersion for RNA-seq data with DESeq2. Genome Biol 2014, 15(12):550.

19. Shen S, Park JW, Lu ZX, Lin L, Henry MD, Wu YN, Zhou Q, Xing Y: rMATS: robust and flexible detection of differential alternative splicing from replicate RNA-Seq data. Proc Natl Acad Sci U SA 2014, 111(51):E5593-5601.

20. Kim D, Salzberg SL: TopHat-Fusion: an algorithm for discovery of novel fusion transcripts. Genome Biology 2011, 12(8).

21. Gao Y, Wang JF, Zhao FQ: CIRl: an efficient and unbiased algorithm for de novo circular RNA identification. Genome Biology 2015, 16.

22. Glazar P, Papavasileiou P, Rajewsky N: circBase: a database for circular RNAs. Rna 2014, 20(11):1666-1670.

23. Szklarczyk D, Gable AL, Lyon D, Junge A, Wyder S, Huerta-Cepas J, Simonovic M, Doncheva NT, Morris JH, Bork P et al: STRING v11: protein-protein association networks with increased coverage, supporting functional discovery in genome-wide experimental datasets. Nucleic Acids Research 2019, 47(D1):D607-D613.

24. Li JH, Liu S, Zhou H, Qu LH, Yang JH: starBase v2.0: decoding miRNA-ceRNA, miRNA-ncRNA and protein-RNA interaction networks from large-scale CLIP-Seq data. Nucleic Acids Research 2014, 42(D1):D92-D97.

25. Shannon P, Markiel A, Ozier O, Baliga NS, Wang JT, Ramage D, Amin N, Schwikowski B, Ideker T: Cytoscape: A software environment for integrated models of biomolecular interaction networks. Genome Res 2003, 13(11):2498-2504.

26. Subramanian A, Tamayo P, Mootha VK, Mukherjee S, Ebert BL, Gillette MA, Paulovich A, Pomeroy SL, Golub TR, Lander ES et al: Gene set enrichment analysis: A knowledge-based approach for interpreting genome-wide expression profiles. P Natl Acad Sci USA 2005, 102(43):15545-15550.

27. Yu GC, Wang LG, Han YY, He QY: clusterProfiler: an R Package for Comparing Biological Themes Among Gene Clusters. Omics 2012, 16(5):284-287.

28. Lee Y, Rio DC: Mechanisms and Regulation of Alternative PremRNA Splicing. Annu Rev Biochem 2015, 84:291-323. 
29. Chibon F, Lagarde P, Salas S, Perot G, Brouste V, Tirode F, Lucchesi C, de Reynies A, Kauffmann A, Bui $B$ et al: Validated prediction of clinical outcome in sarcomas and multiple types of cancer on the basis of a gene expression signature related to genome complexity. Nat Med 2010, 16(7):781-U781.

30. Yen CC, Yeh CN, Cheng CT, Jung SM, Huang SC, Chang TW, Jan YY, Tzeng CH, Chao TC, Chen YY et al: Integrating Bioinformatics and Clinicopathological Research of Gastrointestinal Stromal Tumors: Identification of Aurora Kinase A as a Poor Risk Marker. Ann Surg Oncol 2012, 19(11):3491-3499.

31. Dvinge $H$, Kim E, Abdel-Wahab O, Bradley RK: RNA splicing factors as oncoproteins and tumour suppressors. Nature Reviews Cancer 2016, 16(7):413-430.

32. Kawai A, Woodruff J, Healey JH, Brennan MF, Antonescu CR, Ladanyi M: SYT-SSX gene fusion as a determinant of morphology and prognosis in synovial sarcoma. New Engl J Med 1998, 338(3):153160.

33. Pierron G, Tirode F, Lucchesi C, Reynaud S, Ballet S, Cohen-Gogo S, Perrin V, Coindre JM, Delattre O: A new subtype of bone sarcoma defined by BCOR-CCNB3 gene fusion. Nat Genet 2012, 44(4):461U151.

34. Li WS, Liao IC, Wen MC, Lan HHC, Yu SC, Huang HY: BCOR-CCNB3-positive soft tissue sarcoma with round-cell and spindle-cell histology: a series of four cases highlighting the pitfall of mimicking poorly differentiated synovial sarcoma. Histopathology 2016, 69(5):792-801.

35. Kao YC, Owosho AA, Sung YS, Zhang L, Fujisawa Y, Lee JC, Wexler L, Argani P, Swanson D, Dickson $\mathrm{BC}$ et al: BCOR-CCNB3 Fusion Positive Sarcomas A Clinicopathologic and Molecular Analysis of 36 Cases With Comparison to Morphologic Spectrum and Clinical Behavior of Other Round Cell Sarcomas. Am J Surg Pathol 2018, 42(5):604-615.

36. Pavanello L, Hall B, Airhihen B, Winkler GS: The central region of CNOT1 and CNOT9 stimulates deadenylation by the Ccr4-Not nuclease module. Biochem $J$ 2018, 475:3437-3450.

37. Baranello L, Wojtowicz D, Cui KR, Devaiah BN, Chung HJ, Chan-Salis KY, Guha R, Wilson K, Zhang XH, Zhang HL et al: RNA Polymerase II Regulates Topoisomerase 1 Activity to Favor Efficient Transcription. Cell 2016, 165(2):357-371.

38. Weinstein JN, Collisson EA, Mills GB, Shaw KRM, Ozenberger BA, Ellrott K, Shmulevich I, Sander C, Stuart JM, Network CGAR: The Cancer Genome Atlas Pan-Cancer analysis project. Nat Genet 2013, 45(10):1113-1120.

39. Zhang MC, Xin Y: Circular RNAs: a new frontier for cancer diagnosis and therapy. J Hematol Oncol 2018, 11.

40. Memczak S, Jens M, Elefsinioti A, Torti F, Krueger J, Rybak A, Maier L, Mackowiak SD, Gregersen LH, Munschauer $\mathrm{M}$ et al: Circular RNAs are a large class of animal RNAs with regulatory potency. Nature 2013, 495(7441):333-338.

41. Zhang Y, Zhang XO, Chen T, Xiang JF, Yin QF, Xing YH, Zhu SS, Yang L, Chen LL: Circular Intronic Long Noncoding RNAs. Molecular Cell 2013, 51(6):792-806.

42. Barrett SP, Salzman J: Circular RNAs: analysis, expression and potential functions. Development 2016, 143(11):1838-1847. 
43. Kulcheski FR, Christoff AP, Margis R: Circular RNAs are miRNA sponges and can be used as a new class of biomarker. J Biotechnol 2016, 238:42-51.

44. Panda AC: Circular RNAs Act as miRNA Sponges. Circular Rnas: Biogenesis and Functions 2018, 1087:67-79.

45. Scotti MM, Swanson MS: RNA mis-splicing in disease. Nat Rev Genet 2016, 17(1):19-32.

46. Vaquerizas JM, Kummerfeld SK, Teichmann SA, Luscombe NM: A census of human transcription factors: function, expression and evolution. Nat Rev Genet 2009, 10(4):252-263.

47. Lambert SA, Jolma A, Campitelli LF, Das PK, Yin Y, Albu M, Chen X, Taipale J, Hughes TR, Weirauch MT: The Human Transcription Factors. Cell 2018, 172(4):650-665.

48. Anczukow O, Krainer AR: Splicing-factor alterations in cancers. Rna 2016, 22(9):1285-1301.

49. Zhang ZR, Yang TT, Xiao JJ: Circular RNAs: Promising Biomarkers for Human Diseases. Ebiomedicine 2018, 34:267-274.

50. Haddad G, Lorenzen JM: Biogenesis and Function of Circular RNAs in Health and in Disease. Front Pharmacol 2019, 10.

\section{Figures}


A

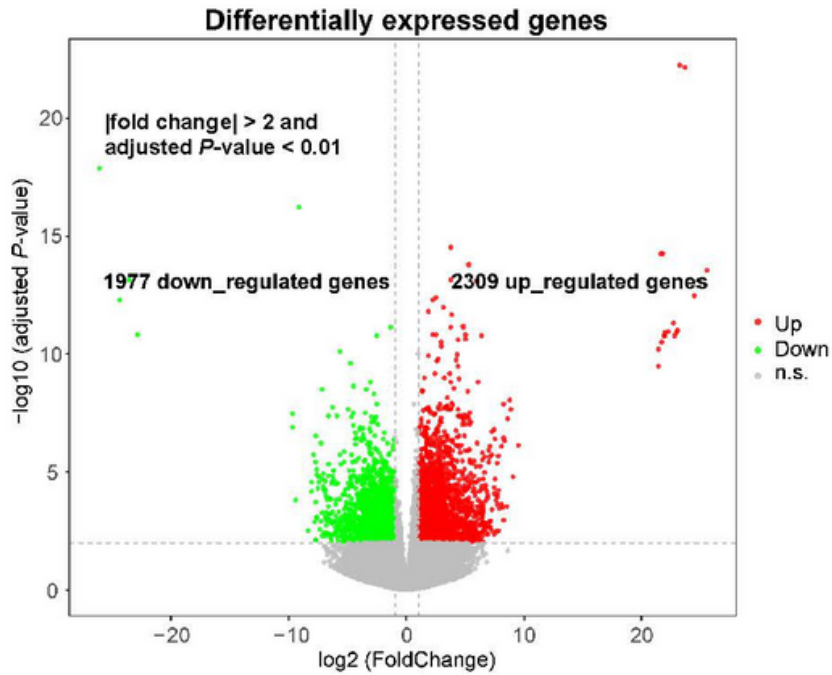

c

Enriched biological processes

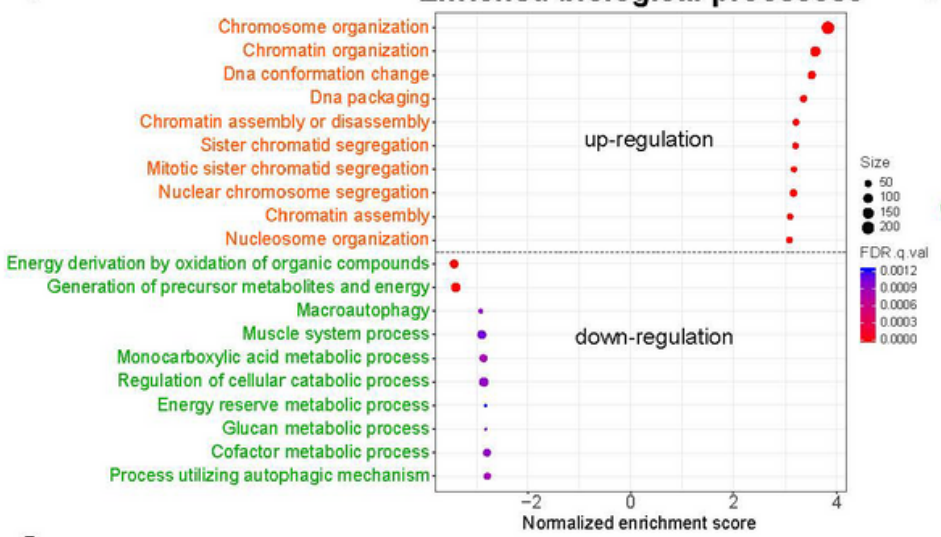

E

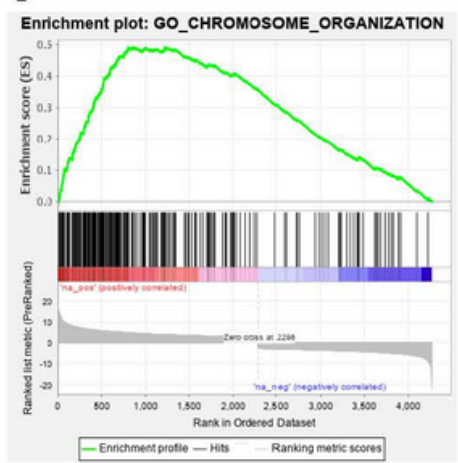

B

Four categories of differentially expressed genes (590 out of 4286 DEGs)
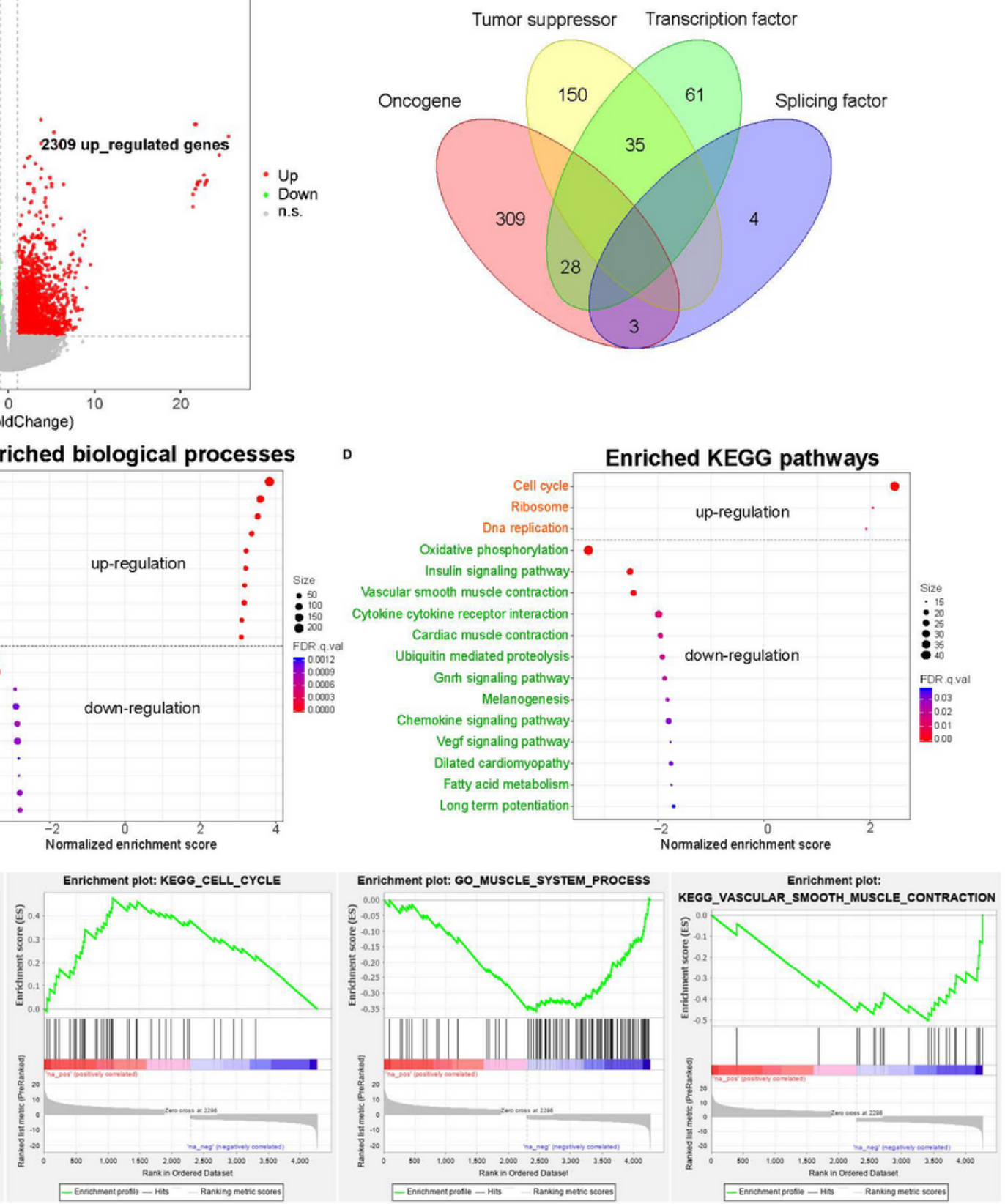

\section{Figure 1}

Differential expression profile and corresponding gene functions of SS. A. Volcano plot displaying differentially expressed genes (DEGs) between ten pairs of SS and tumor-adjacent tissues. |fold change| $>2$ and adjusted P-value $<0.01$. B. Different categories of DEGs. C. Top enriched up-regulated and downregulated biological processes of DEGs. D. Top enriched up-regulated and down-regulated KEGG pathways for DEGs. E. Examples of enriched biological processes and pathways. Adjusted P-value $<0.05$. 

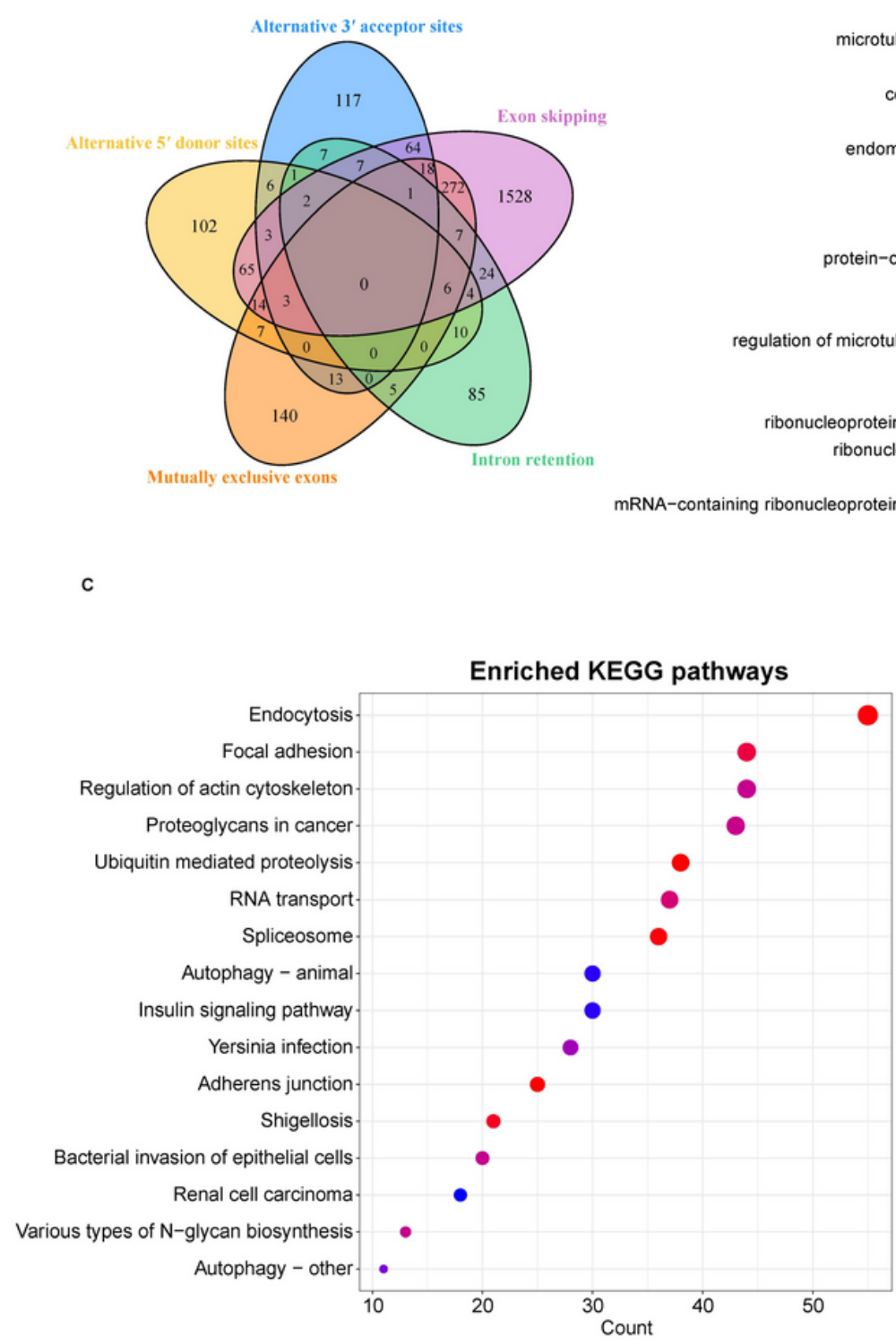

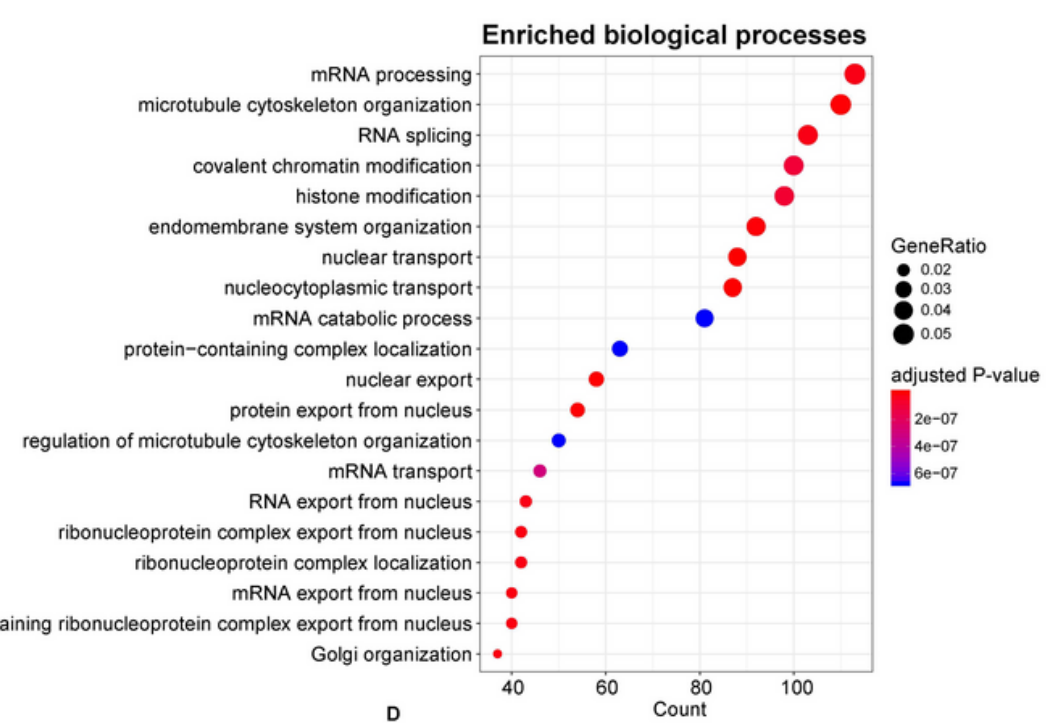

Four categories of differential alternative splicing genes ( 563 out of 2511 DASGs)

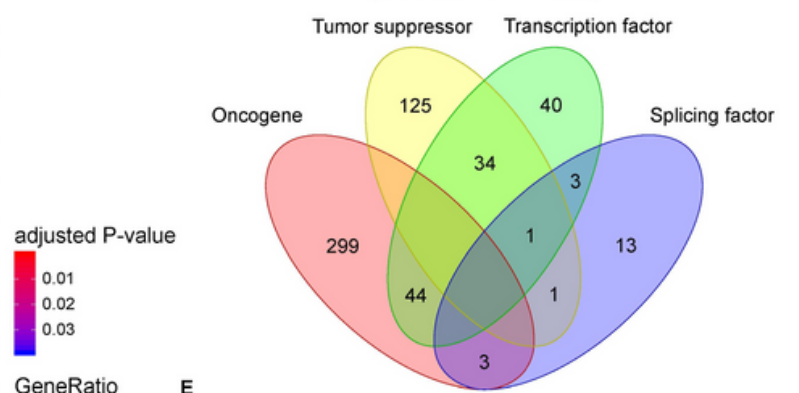

GeneRatio E

0.02

0.03

0.04

04

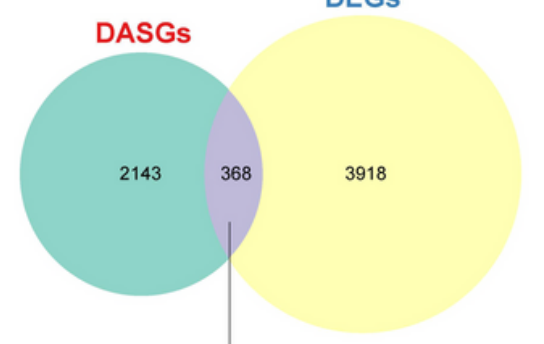

Enriched biological process : Actomyosin structure organization, adjusted P-value $=0.034$ Enriched KEGG pathway : Regulation of actin cytoskeleton, adjusted $P$-value $=0.0425$

Figure 2

Alternative splicing patterns and related gene functions of SS. A. Venn plot of the differentially alternative spliced genes (DASGs) for five common splicing modes. B. Top 20 enriched biological processes of DASGs. FDR < 0.05. C. Top 20 enriched pathways for DASGs. Adjusted P-value < 0.05. D. Distinct categories of DASGs. E. Comparison between DEGs and DASGs. 

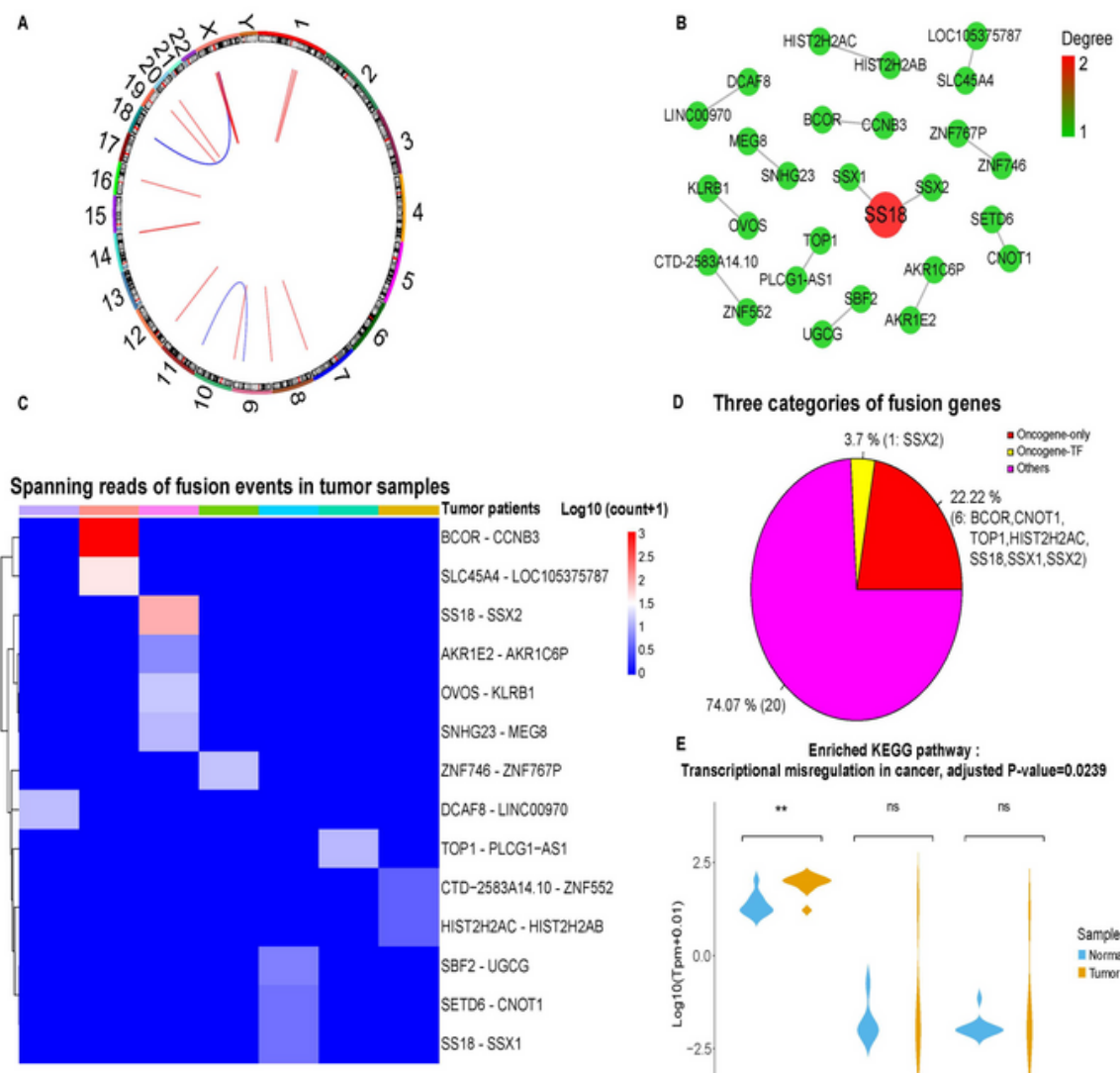

D Three categories of fusion genes

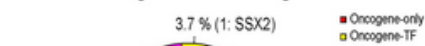
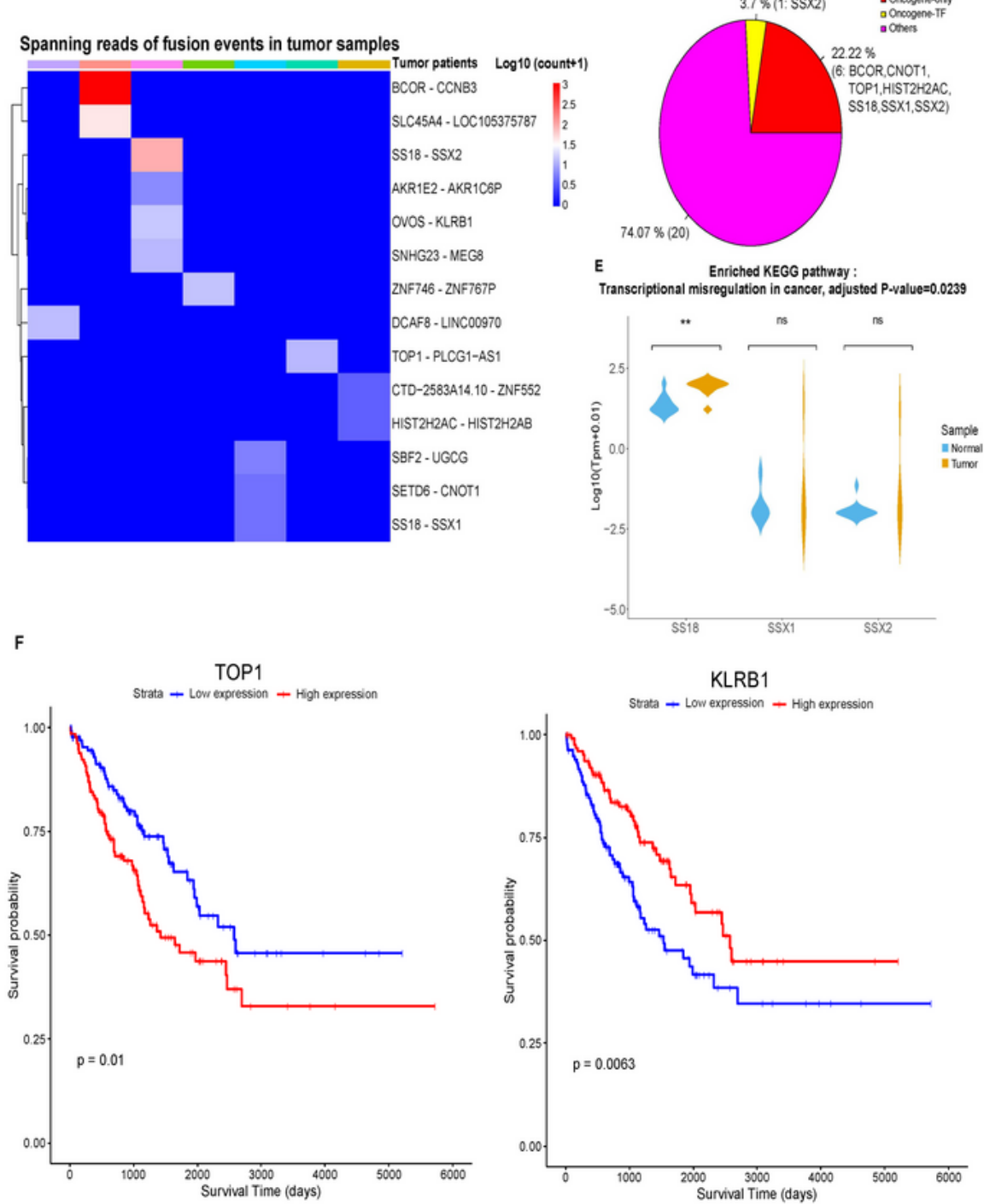

\section{Figure 3}

Gene fusion landscape of SS. A. Circos plot showing the 14 tumor-specific fusion pairs in SS. B. Network of tumor-specific fusion genes. The size and color of each circle correspond to the degree of fusion edges. C. Heatmap displaying the supporting junction reads for tumor-specific gene fusions. D. Different categories of the fusion genes. E. Expression profile of the fusion genes involved in significantly enriched 
biological processes. Adjusted P-value $<0.05$. F. Kaplan - Meier survival analysis for fusion genes based on the TCGA sarcoma data. P-value $<0.05$.
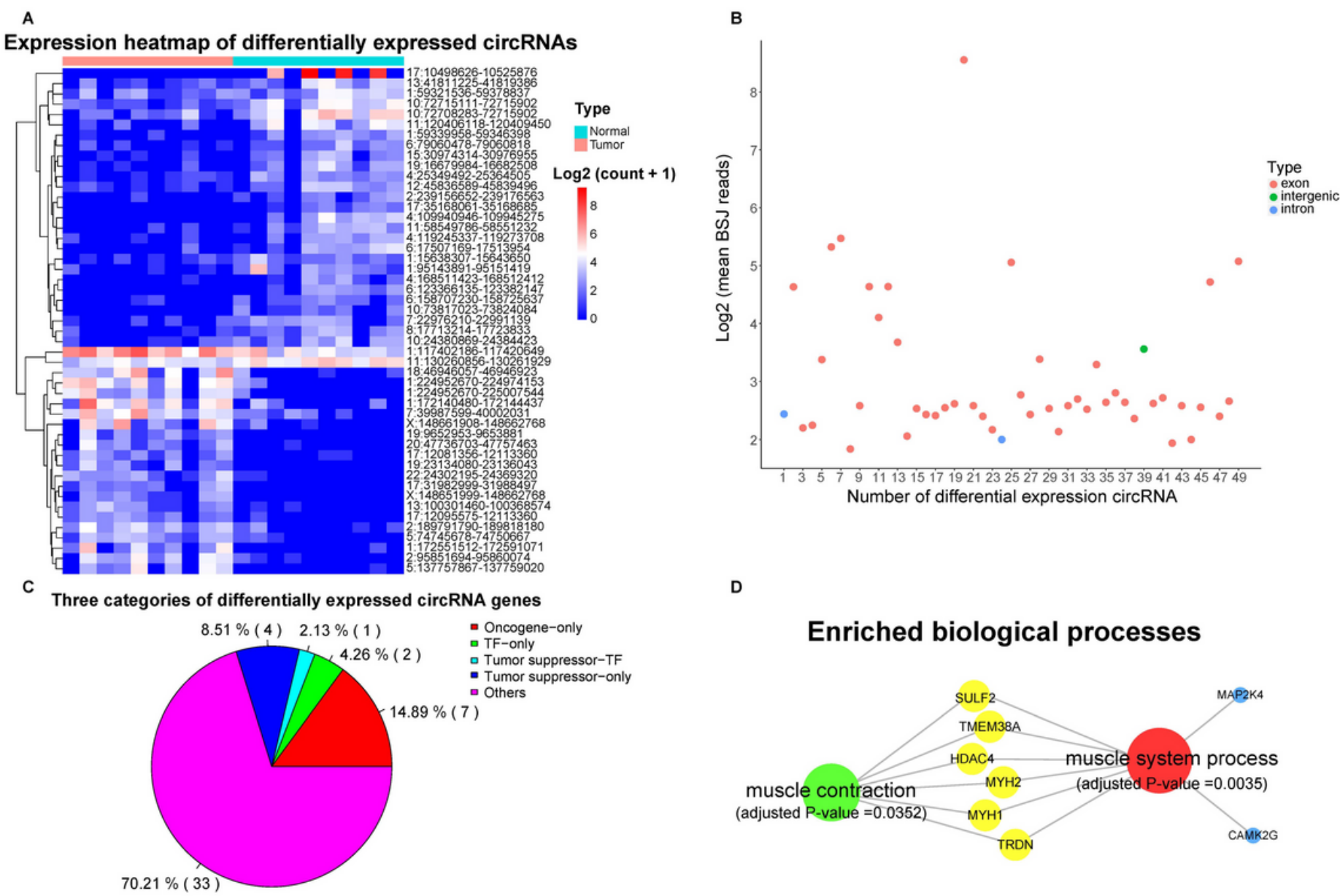

D

\section{Enriched biological processes}

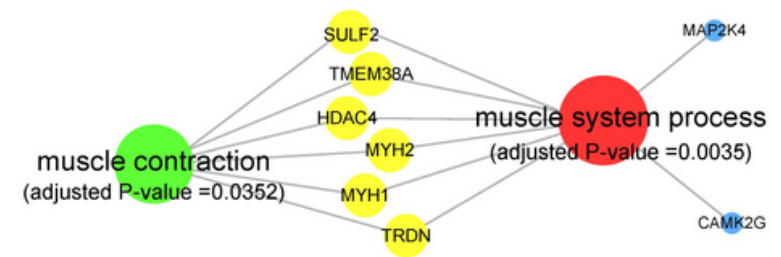

\section{Figure 4}

Expression profile and characteristics of circRNAs. A. Expression heatmap showing the differentially expressed circRNAs. Supporting reads of circRNAs were used in heatmap. B. Genomic region of the differentially expressed circRNAs. BSJ: back-spliced junction. C. Distinct types of the parental genes of differentially expressed circRNAs. D. Significantly enriched biological processes for the parental genes of differentially expressed circRNAs. Adjusted P-value $<0.05$. 
DNM3OS, ZNF730, DNAH14, AFF2, TMEM38A, TMEM56,CAP2, SYTL3, MCU, KATNAL2

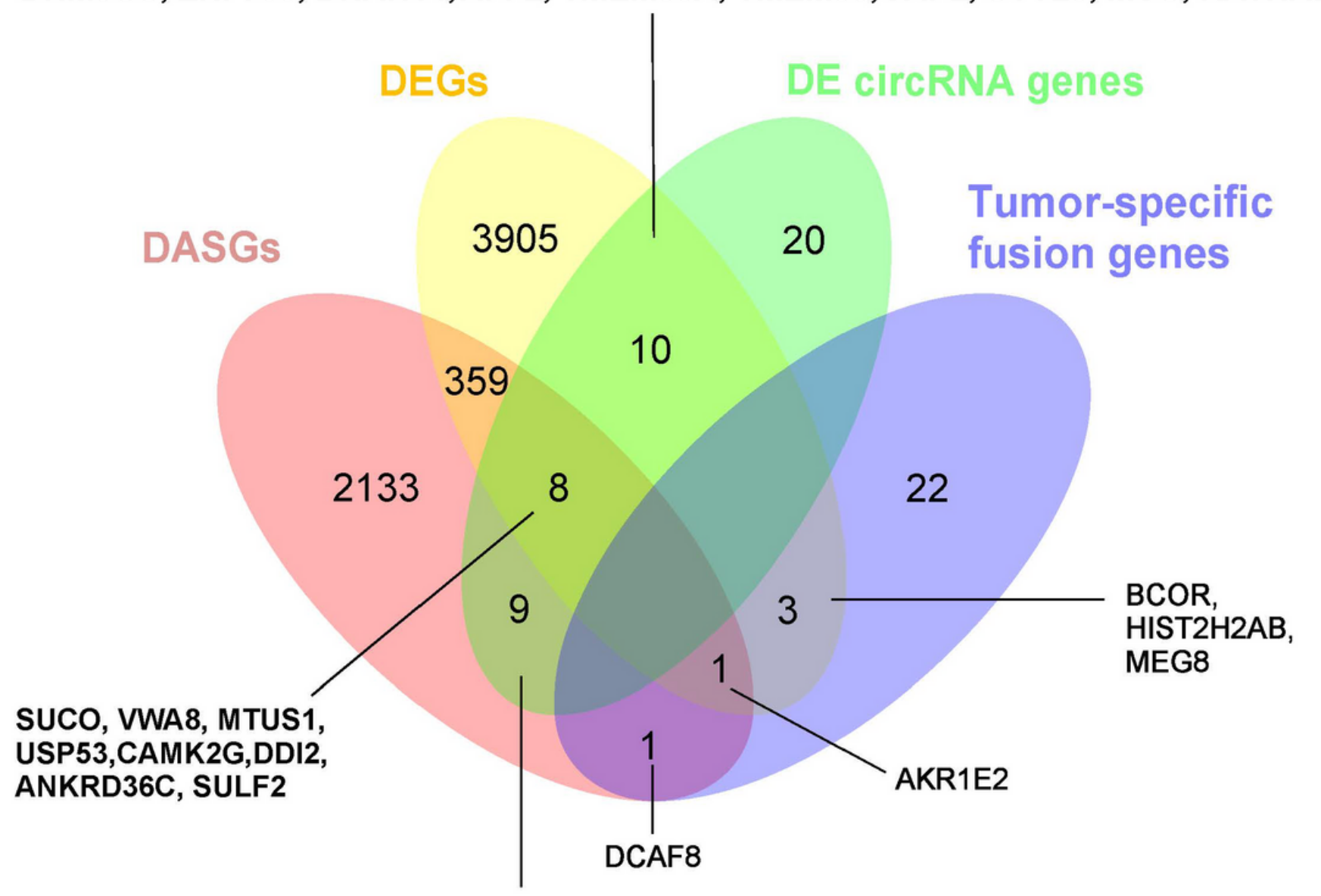

PMS1, MAP2K4, HDAC4, KIAA1217, PALLD, ZNF562, FGGY, ARHGEF12, SPECC1L-ADORA2A

Figure 5

Comparison of DEGs, DASGs, fusion genes, and the parental genes of differentially expressed circRNAs. The overlapped genes for each part were shown on the figure. 


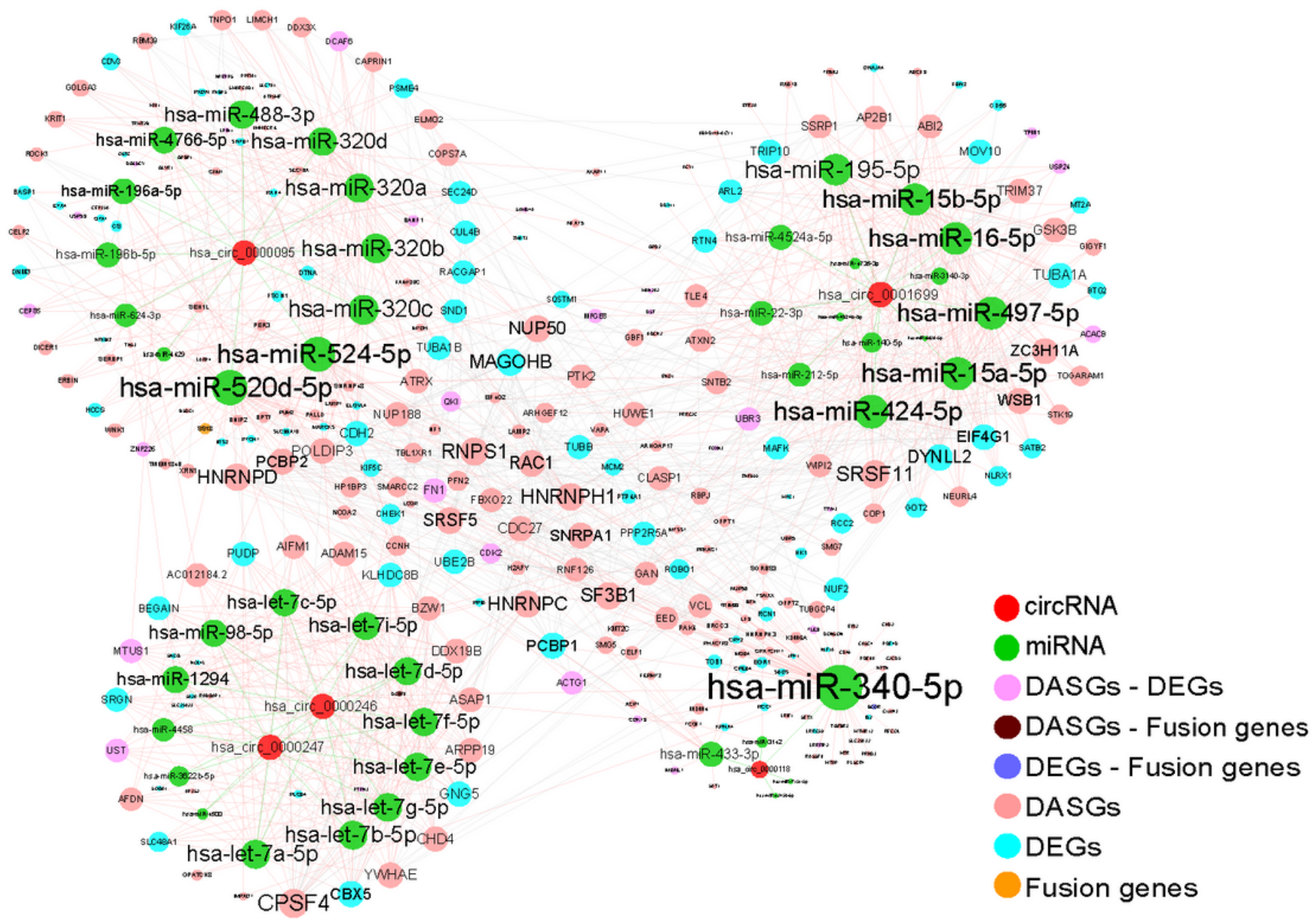

Figure 6

Interaction network among circRNAs, miRNAs, and related target genes. The size of each node is in proportion to the degree of edges. Only the target genes of DEGs, DASGs, fusion genes, and the parental genes of differentially expressed circRNAs were considered for corresponding miRNAs. The proteinprotein interactions were obtained from the STRING database, while the known circRNA -miRNA interactions were downloaded from starBase.

\section{Supplementary Files}

This is a list of supplementary files associated with this preprint. Click to download.

- SupplementaryFigureS1.tiff

- SupplementaryFigureS2.tiff

- SupplementaryfigureS3.tiff

- SupplementaryTableS1.csv

- SupplementaryTableS2.csv 
- SupplementaryFileS1.pdf

Page 22/22 